\title{
Dynamics of Stochastically Perturbed SIS Epidemic Model with Vaccination
}

\author{
Yanan Zhao ${ }^{1,2}$ and Daqing Jiang ${ }^{1}$ \\ ${ }^{1}$ School of Mathematics and Statistics, Northeast Normal University, Changchun, Jilin 130024, China \\ ${ }^{2}$ School of Science, Changchun University, Changchun, Jilin 130022, China
}

Correspondence should be addressed to Daqing Jiang; daqingjiang2010@hotmail.com

Received 2 February 2013; Revised 12 July 2013; Accepted 12 July 2013

Academic Editor: Julio Rossi

Copyright ( 2013 Y. Zhao and D. Jiang. This is an open access article distributed under the Creative Commons Attribution License, which permits unrestricted use, distribution, and reproduction in any medium, provided the original work is properly cited.

\begin{abstract}
We introduce stochasticity into an SIS epidemic model with vaccination. The stochasticity in the model is a standard technique in stochastic population modeling. In the deterministic models, the basic reproduction number $R_{0}$ is a threshold which determines the persistence or extinction of the disease. When the perturbation and the disease-related death rate are small, we carry out a detailed analysis on the dynamical behavior of the stochastic model, also regarding of the value of $R_{0}$. If $R_{0} \leq 1$, the solution of the model is oscillating around a steady state, which is the disease-free equilibrium of the corresponding deterministic model, whereas, if $R_{0}>1$, there is a stationary distribution, which means that the disease will prevail. The results are illustrated by computer simulations.
\end{abstract}

\section{Introduction}

Epidemiology is the study of the spread of diseases with the objective to trace factors that are responsible for or contribute to their occurrence. Significant progress has been made in the theory and application of epidemiology modeling by mathematical research. Controlling infectious diseases has been an increasingly complex issue in recent years. Vaccination is an important strategy for the elimination of infectious diseases [1-3]. The vaccination enables the vaccinated to acquire a permanent or temporary immunity. When the immunity is temporary, the immunity can be lost after a period of time. This temporary immunity is used in many references [47] that assume the process of losing immunity is in the exponential form.

In [7], Li and Ma discussed an SIS model with vaccination. The system has following form:

$$
\begin{gathered}
\dot{S}(t)=(1-q) A-\beta S(t) I(t)-(\mu+p) S(t) \\
+\gamma I(t)+\varepsilon V(t), \\
\dot{I}(t)=\beta S(t) I(t)-(\mu+\gamma+a) I(t), \\
\dot{V}(t)=q A+p S(t)-(\mu+\varepsilon) V(t) .
\end{gathered}
$$

Here $S(t)$ denotes the number of members of a population who are susceptible to an infection at time $t . I(t)$ denotes the number of members who are infective at time $t . V(t)$ denotes the number of members who are immune to an infection at time $t$ as the result of vaccination. The derivatives $d S(t) / d t$, $d I(t) / d t$, and $d V(t) / d t$ are denoted by $\dot{S}(t), \dot{I}(t)$, and $\dot{V}(t)$, respectively.

The parameters in the model are summarized in the following list:

$A$ : a constant input of new members into the population per unit time;

$q$ : a fraction of vaccinated for newborns;

$\beta$ : transmission coefficient between compartments $S$ and $I$;

$\mu$ : natural death rate of $S, I$, and $V$ compartments;

$p$ : the proportional coefficient of vaccinated for the susceptible;

$\gamma$ : recovery rate of infectious individuals;

$\varepsilon$ : the rate of losing their immunity for vaccinated individuals;

$\alpha$ : disease-caused death rate of infectious individuals. 
All parameter values are assumed to be nonnegative and $\mu, A>0$.

In $\mathrm{Li}$ and $\mathrm{Ma}$ [7], system (1) always has the disease-free equilibrium $P_{0}=\left(S_{0}, I_{0}, V_{0}\right)=((A / \mu)((\mu(1-q)+\varepsilon) /(\mu+$ $\varepsilon+p)), 0,(A / \mu)((\mu q+p) /(\mu+\varepsilon+p)))$. If $R_{0} \leqslant 1$, then $P_{0}$ is the unique equilibrium of (1), and it is globally stable in the invariant set $\Gamma$, where $\Gamma=\{(S, I, V): S>0, I \geq$ $0, V \geq 0, S+I+V \leq A / \mu\}$. If $R_{0}>1$, then $P_{0}$ is unstable and there is an endemic equilibrium $P^{*}=\left(S^{*}, I^{*}, V^{*}\right)=$ $\left((\mu+\gamma+\alpha) / \beta,(\mu(\mu+\gamma+\alpha)(\mu+\varepsilon+p) / \beta(\mu+\alpha)(\mu+\varepsilon))\left(R_{0}-\right.\right.$ $1),(q A+(p / \beta)(\mu+\gamma+\alpha)) /(\mu+\varepsilon))$ of (1), which is globally asymptotically stable under the sufficient condition in the invariant set $\Gamma$. The basic reproduction number of system (1) is as follows:

$$
R_{0}=\frac{A \beta(\mu(1-q)+\varepsilon)}{\mu(\mu+\gamma+\alpha)(\mu+\varepsilon+p)}=\frac{\beta S_{0}}{\mu+\gamma+\alpha} .
$$

Considering real life is full of randomness and stochasticity, using stochastic models can gain more real benefits. Consequently, there are many authors who studied stochastic biological systems and stochastic epidemic models; see [824]. There may be different approaches which result in different effects on the population system to include random perturbations in the models. In [20-23], the situation of the parameter perturbation was considered. Gray et al. [23] discuss the following stochastic SIS model:

$$
\begin{aligned}
d S(t)= & {[\mu N-\beta S(t) I(t)+\gamma I(t)-\mu S(t)] d t } \\
& -\sigma S(t) I(t) d B(t), \\
d I(t)= & {[\beta S(t) I(t)-(\mu+\gamma) I(t)] d t } \\
& +\sigma S(t) I(t) d B(t) .
\end{aligned}
$$

Here $S$ and $I$ denote the susceptible and infected numbers of individuals, respectively. With $S(t)+I(t) \equiv N$, they simplify system (3) into a single equation and show that if $R_{0}^{S}<1$ and $\sigma^{2} \leq \beta / N$, then the disease will die out with probability one. If $R_{0}^{S}>1$, then the disease will be persistent, where $R_{0}^{S}=R_{0}^{D-}$ $\left(\sigma^{2} N^{2} / 2(\mu+\gamma)\right)$ and $R_{0}^{D}=\beta N /(\mu+\gamma)$ is the threshold of the corresponding deterministic system. $R_{0}^{S}$ can be considered as the threshold of system (3), which is less than the value of $R_{0}^{D}$.

On the other hand, white noise stochastic perturbations around the positive endemic equilibrium of epidemic models were considered in $[19,25]$. Beretta et al. [25] considered the following system differential equation:

$$
\begin{aligned}
d S(t)= & -\beta S(t) \int_{0}^{h} f(s) I(t-s) d s-\mu_{1} S(t)+b \\
& +\sigma_{1}\left(S(t)-S^{*}\right) d B_{1}(t), \\
d I(t)= & \beta S(t) \int_{0}^{h} f(s) I(t-s) d s-\left(\mu_{2}+\lambda\right) I(t) \\
& +\sigma_{2}\left(I(t)-I^{*}\right) d B_{2}(t), \\
d R(t)= & \lambda I(t)-\mu_{3} R(t)+\sigma_{3}\left(R(t)-R^{*}\right) d B_{3}(t),
\end{aligned}
$$

where $S^{*}, I^{*}$, and $R^{*}$ are the positive points of equilibrium for the corresponding deterministic system and $\sigma_{i}$ are constants, $B_{i}(t)$ are independent from other standard Wiener processes $(i=1,2,3)$. In their paper, they proved the above stochastic system was stable in probability by using the general method of Lyapunov functionals construction and obtained the stability condition immediately in terms of the parameters of the system under consideration.

Another different approach to include stochastic perturbations in a biological model was considered by Imhof and Walcher in [11]. They introduced and analyzed a variant of the deterministic single-substrate chemostat model. Also they found all species were persistent in the multiple species by using a comparison principle. Subsequently, they turned to modeling the influence of random fluctuations on the deterministic chemostat model. They set up the following stochastic chemostat model:

$$
\begin{aligned}
d X_{0}= & \left(r-\delta X_{0}-a_{1}\left(X_{0}, X_{1}\right)-a_{2}\left(X_{0}, X_{2}\right)\right) d t \\
& +\sigma_{0} X_{0} d B_{0}(t) \\
d X_{1}= & \left(a_{1}\left(X_{0}, X_{1}\right)-s_{1}\left(X_{1}, X_{2}\right)\right) d t \\
& +\sigma_{1} X_{1} d B_{1}(t) \\
d X_{2}= & \left(a_{2}\left(X_{0}, X_{2}\right)-s_{2}\left(X_{1}, X_{2}\right)\right) d t \\
& +\sigma_{2} X_{2} d B_{1}(t)
\end{aligned}
$$

where $\sigma_{0}, \sigma_{1}, \sigma_{2} \geq 0$ and $B_{i}(t)(i=1,2,3)$ are independent Brownian motions. By analyzing this stochastic differential equation model, they proved that the stochastic model led to extinction even though the deterministic counterpart predicts persistence.

In this paper, our approach to include stochastic perturbation is analogous to that of Imhof and Walcher [11]. We are devoted to studying the following stochastic system:

$$
\begin{gathered}
d S(t)=[(1-q) A-\beta S I-(\mu+p) S+\gamma I+\varepsilon V] d t \\
+\sigma_{1} S d B_{1}(t), \\
d I(t)=[\beta S-(\mu+\gamma+\alpha)] I d t+\sigma_{2} I d B_{2}(t), \\
d V(t)=[q A+p S-(\mu+\varepsilon) V] d t+\sigma_{3} V d B_{3}(t),
\end{gathered}
$$

where $B_{i}(t)(i=1,2,3)$ are independent Brownian motions and $\sigma_{i}(i=1,2,3)$ are their intensities.

This paper is organized as follows. In Section 2, we show there is a unique positive solution of system (6) by the way mentioned in $[20,23]$. In Section 3 , when $R_{0} \leq 1$, we derive that the solution of the system (6) oscillates around the disease-free equilibrium $P_{0}$ of system (1). In Section 4, when $R_{0}>1$, although the solution of system (6) does not converge to the endemic proportion equilibrium $P^{*}$ of system (1), we conclude there is a unique stationary distribution for system (6), and it has ergodic property under some conditions. In Section 5, we make simulations to confirm our analytical results. Finally, in order to be self-contained, we have the appendix. 


\section{Existence and Uniqueness of Positive Solution}

To investigate the dynamical behavior, the first concern is whether the solution has a global existence. Moreover, for a population dynamics model, whether the value is nonnegative is also considered. Hence in this section, we first show that the solution of system (6) is global and nonnegative.

Theorem 1. There is a unique solution $Y(t)=(S(t), I(t), V(t))$ of system (6) on $t \geqslant 0$ for any initial value $Y(0)=$ $(S(0), I(0), V(0)) \in \mathbb{R}_{+}^{3}$, and the solution will remain in $\mathbb{R}_{+}^{3}$ with probability 1 , namely, $Y(t) \in \mathbb{R}_{+}^{3}$ for all $t \geqslant 0$ almost surely.

Proof. Our proof is motivated by the works of Mao et al. [17]. Since the coefficients of (6) are locally Lipschitz continuous for any given initial value $(S(0), I(0), V(0)) \in \mathbb{R}_{+}^{3}$, there is a unique local solution $(S(t), I(t), V(t))$ on $t \in\left[0, \tau_{e}\right)$, where $\tau_{e}$ is the explosion time (see [20]). To show that this solution is global, we need to show that $\tau_{e}=\infty$ a.s. Let $k_{0} \geq 0$ be sufficiently large so that $S(0), I(0)$, and $V(0)$ all lie within the interval $\left[1 / k_{0}, k_{0}\right]$. For each integer $k \geqslant k_{0}$, define the stopping time:

$$
\begin{aligned}
\tau_{k}=\inf \{t & \in\left[0, \tau_{e}\right): \min \{S(t), I(t), V(t)\} \\
\leq & \left.\frac{1}{k} \text { or } \max \{S(t), I(t), V(t)\} \geq k\right\},
\end{aligned}
$$

where throughout this paper, we set inf $\emptyset=\infty$ (as usual $\emptyset$ denotes the empty set). According to the definition, $\tau_{k}$ is increasing as $k \rightarrow \infty$. Set $\tau_{\infty}=\lim _{k \rightarrow \infty} \tau_{k}$, whence $\tau_{\infty} \leqslant \tau_{e}$ a.s. If we can show that $\tau_{\infty}=\infty$ a.s., then $\tau_{e}=\infty$ and $(S(t), I(t), V(t)) \in \mathbb{R}_{+}^{3}$ a.s. for all $t \geqslant 0$. In other words, to complete the proof, all we need to show is that $\tau_{\infty}=\infty$ a.s. If this statement is false, then there exist a pair of constants $T>0$ and $\varepsilon \in(0,1)$ such that

$$
P\left\{\tau_{\infty} \leqslant T\right\}>\varepsilon
$$

Hence there is an integer $k_{1} \geqslant k_{0}$ such that

$$
P\left\{\tau_{k} \leqslant T\right\} \geqslant \varepsilon \quad \forall k \geqslant k_{1}
$$

Define a $C^{2}$-function $W: \mathbb{R}_{+}^{3} \rightarrow \overline{\mathbb{R}}$ (see the appendix) by

$$
\begin{aligned}
W= & \left(S-a-a \log \frac{S}{a}\right)+(I-1-\log I) \\
& +(V-1-\log V) .
\end{aligned}
$$

The nonnegativity of this function can be seen from $u-1-$ $\log u \geqslant 0$, for all $u>0$. Let $k \geqslant k_{0}$ and $T>0$ be arbitrary. Applying the Itô formula, we obtain

$$
\begin{aligned}
d W= & \left(1-\frac{a}{S}\right)[(1-q) A-\beta S I-(\mu+p) S+\gamma I+\varepsilon V] d t \\
& +\left(1-\frac{a}{S}\right) \sigma_{1} S d B_{1}(t)+\frac{1}{2} a \sigma_{1}^{2} d t \\
& +\left(1-\frac{1}{I}\right)[\beta S-(\mu+\gamma+\gamma)] I d t \\
& +\left(1-\frac{1}{I}\right) \sigma_{2} I d B_{2}(t)+\frac{1}{2} \sigma_{2}^{2} d t \\
& +\left(1-\frac{1}{V}\right)[q A+p S-(\mu+\varepsilon) V] d t \\
& +\left(1-\frac{1}{V}\right) \sigma_{3} V d B_{3}(t)+\frac{1}{2} \sigma_{3}^{2} d t \\
= & L W d t+\sigma_{1}(S-a) d B_{1}(t) \\
& +\sigma_{2}(I-1) d B_{2}(t)+\sigma_{3}(V-1) d B_{3}(t),
\end{aligned}
$$

where $L W: \mathbb{R}_{+}^{3} \rightarrow \mathbb{R}_{+}$is defined by

$$
\begin{aligned}
L W(S, I, V)= & A-\mu S-(1-q) A \frac{a}{S}+a \beta I+a(\mu+p) \\
& -a \gamma \frac{I}{S}-a \varepsilon \frac{V}{S}+\frac{1}{2} a \sigma_{1}^{2}-(\mu+\alpha) I \\
& -\beta S+(\mu+\alpha+\gamma)+\frac{1}{2} \sigma_{2}^{2}-\mu V \\
& -q \frac{A}{V}-p \frac{S}{V}+(\mu+\varepsilon)+\frac{1}{2} \sigma_{3}^{2} \\
\leqslant & A+[a \beta-(\mu+\alpha)] I+a(\mu+p) \\
& +\frac{1}{2} a \sigma_{1}^{2}+\frac{1}{2} \sigma_{2}^{2}+\frac{1}{2} \sigma_{3}^{2} \\
& +(\mu+\alpha+\gamma)+(\mu+\varepsilon) .
\end{aligned}
$$

Let $a=(\mu+\alpha) / \beta$, s.t. $a \beta-(\mu+\alpha)=0$. Substituting this into (12), we get

$$
\begin{aligned}
L W(S, I, V) \leqslant & A+\frac{(\mu+\alpha)(\mu+p)}{\beta}+\frac{\mu+\alpha}{2 \beta} \sigma_{1}^{2} \\
& +(\mu+\gamma+\alpha)+\frac{1}{2} \sigma_{2}^{2} \\
& +(\mu+\varepsilon)+\frac{1}{2} \sigma_{3}^{2} \\
:= & K .
\end{aligned}
$$

The remainder of the proof follows that in Mao et al. [17].

\section{Asymptotic Behavior around $P_{0}$}

It is clear that $P_{0}=\left(S_{0}, 0, V_{0}\right)$ is the disease-free equilibrium of system (1). If $R_{0} \leq 1$, then $P_{0}$ is globally stable, which 
means the disease will die out after some period of time. Hence, it is interesting to study the disease-free equilibrium for controlling infectious disease. But there is no disease-free equilibrium in system (6). In this section, we show the average oscillation around $P_{0}$ in time to exhibit whether the disease will die out.

Theorem 2. If $R_{0} \leqslant 1, \alpha^{2}<4 \mu(\mu+\alpha)(1+2 \mu / \varepsilon)$, and the following condition is satisfied

$$
\begin{gathered}
\mu\left(\frac{2(\mu+p)}{\varepsilon}+1\right)>\left(c_{2}+1\right) \sigma_{1}^{2}, \\
(\mu+\alpha)\left(1+\frac{2 \mu}{\varepsilon}\right)-\frac{\alpha}{4 \rho}>\left(c_{2}+1\right) \sigma_{2}^{2}, \\
(\mu-\rho \alpha)>\sigma_{3}^{2},
\end{gathered}
$$

then the solution $Y(t)$ of system (6) with initial value $Y(0) \in \mathbb{R}_{+}^{3}$ has the property

$$
\begin{aligned}
& \limsup _{t \rightarrow \infty} \frac{1}{t} E \int_{0}^{t}\{ {\left[\mu\left(\frac{2(\mu+p)}{\varepsilon}+1\right)-\left(c_{2}+1\right) \sigma_{1}^{2}\right] } \\
& \times\left(S(r)-S_{0}\right)^{2} \\
&+ {\left[(\mu+\alpha)\left(1+\frac{2 \mu}{\varepsilon}\right)\right.} \\
&\left.-\frac{\alpha}{4 \rho}-\left(c_{2}+1\right) \sigma_{2}^{2}\right] I(r)^{2} \\
&\left.+\left[(\mu-\rho \alpha)-\sigma_{3}^{2}\right]\left(V(r)-V_{0}\right)^{2}\right\} d s \\
& \leqslant\left(c_{2}+1\right) \sigma_{1}^{2} S_{0}^{2}+\sigma_{3}^{2} V_{0}^{2},
\end{aligned}
$$

where $P_{0}=\left(S_{0}, 0, V_{0}\right)$ is the disease-free equilibrium of system (1), $c_{2}=2 \mu / \varepsilon$, and $\rho$ is positive constant that satisfied $\alpha / 4(\mu+$ $\alpha)(1+2 \mu / \varepsilon)<\rho<\mu / \alpha$.

Proof. Let $x=S-S_{0}, y=I$, and $z=V-V_{0}$. Then

$$
\begin{aligned}
d x= & {\left[-\beta\left(x+S_{0}\right) y-(\mu+p) x+\gamma y+\varepsilon z\right] d t } \\
& +\sigma_{1}\left(x+S_{0}\right) d B_{1}(t), \\
d y= & \left\{\beta x y+\left[\beta S_{0}-(\mu+\gamma+\alpha)\right] y\right\} d t \\
& +\sigma_{2} y d B_{2}(t), \\
d z= & {[p x-(\mu+\varepsilon) z] d t } \\
& +\sigma_{3}\left(z+V_{0}\right) d B_{3}(t) .
\end{aligned}
$$

Define a $C^{2}$-function $W: \mathbb{R}_{+}^{3} \rightarrow \mathbb{R}_{+}$by

$$
\begin{aligned}
W(x, y, z) & =c_{1} y+\frac{c_{2}}{2}(x+y)^{2}+\frac{1}{2}(x+y+z)^{2} \\
& :=c_{1} W_{1}(x, y, z)+c_{2} W_{2}(x, y, z)+W_{3}(x, y, z),
\end{aligned}
$$

where $c_{1}=1 / \beta[2 \mu(2 \mu+p+\alpha) / \varepsilon+(2 \mu+\alpha)]$. By Itô's formula, we compute

$$
\left.\begin{array}{rl}
d W_{1}= & \left\{\beta x y+\left[\beta S_{0}-(\mu+\gamma+\alpha)\right] y\right\} d t+\sigma_{2} y d B_{2}(t) \\
= & {\left[\beta x y+(\mu+\gamma+\alpha)\left(R_{0}-1\right) y\right] d t+\sigma_{2} y d B_{2}(t)} \\
= & L W_{1} d t+\sigma_{2} y d B_{2}(t) \\
d W_{2}=(x+y)\{[-(\mu+p) x-(\mu+\alpha) y
\end{array}\right\}
$$

If $R_{0} \leqslant 1$, then

$$
\begin{aligned}
L W= & c_{1} L W_{1}+c_{2} L W_{2}+L W_{3} \\
\leqslant & {\left[c_{1} \beta-c_{2}(2 \mu+p+\alpha)-(2 \mu+\alpha)\right] x y } \\
& -\left[c_{2}(\mu+p)+\mu\right] x^{2} \\
& -\left(c_{2}+1\right)(\mu+\alpha) y^{2}-\mu z^{2} \\
& +\left(c_{2} \varepsilon-2 \mu\right) x z+\left[c_{2} \varepsilon-(2 \mu+\alpha)\right] y z \\
& +\frac{1}{2}\left(c_{2}+1\right) \sigma_{1}^{2}\left(x+S_{0}\right)^{2} \\
& +\frac{1}{2}\left(c_{2}+1\right) \sigma_{2}^{2} y^{2}+\frac{1}{2} \sigma_{3}^{2}\left(z+V_{0}\right)^{2} .
\end{aligned}
$$

Note that

$$
c_{1} \beta-c_{2}(2 \mu+p+\alpha)-(2 \mu+\alpha)=0, \quad c_{2} \varepsilon-2 \mu=0 .
$$


Therefore, we can obtain

$$
\begin{aligned}
L W \leqslant & -\mu\left[\frac{2(\mu+p)}{\varepsilon}+1\right] x^{2} \\
& -\left(1+\frac{2 \mu}{\varepsilon}\right)(\mu+\alpha) y^{2}-\mu z^{2}-\alpha y z \\
+ & \frac{1}{2}\left(c_{2}+1\right) \sigma_{1}^{2}\left(x+S_{0}\right)^{2} \\
+ & \frac{1}{2}\left(c_{2}+1\right) \sigma_{2}^{2} y^{2}+\frac{1}{2} \sigma_{3}^{2}\left(z+V_{0}\right)^{2} \\
\leqslant & -\left\{\mu\left[\frac{2(\mu+p)}{\varepsilon}+1\right]-\left(c_{2}+1\right) \sigma_{1}^{2}\right\} x^{2} \\
& -\left[(\mu+\alpha)\left(1+\frac{2 \mu}{\varepsilon}\right)-\frac{\alpha}{4 \rho}\right. \\
& \left.-\left(c_{2}+1\right) \sigma_{2}^{2}-\frac{\alpha}{4 \rho}\right] y^{2}-\left[(\mu-\rho \alpha)-\sigma_{3}^{2}\right] z^{2} \\
+ & \left(c_{2}+1\right) \sigma_{1}^{2} S_{0}^{2}+\sigma_{3}^{2} V_{0}^{2},
\end{aligned}
$$

where $\rho$ is positive constant to be specified later and Young's inequality is used. If

$$
\alpha^{2}<4 \mu(\mu+\alpha)\left(1+\frac{2 \mu}{\varepsilon}\right)
$$

we choose $\rho$ such that $\alpha / 4(\mu+\alpha)(1+2 \mu / \varepsilon)<\rho<\mu / \alpha$. This implies

$$
(\mu+\alpha)\left(1+\frac{2 \mu}{\varepsilon}\right)-\frac{\alpha}{4 \rho}>0, \quad \mu-\rho \alpha>0
$$

Then we have

$$
\begin{aligned}
d W \leqslant-\left\{\left[\mu\left(\frac{2(\mu+p)}{\varepsilon}+1\right)-\left(c_{2}+1\right) \sigma_{1}^{2}\right] x^{2}\right. \\
+\left[(\mu+\alpha)\left(1+\frac{2 \mu}{\varepsilon}\right)-\frac{\alpha}{4 \rho}-\left(c_{2}+1\right) \sigma_{2}^{2}\right] y^{2} \\
+\left[(\mu-\rho \alpha)-\sigma_{3}^{2}\right] z^{2} \\
\left.\quad-\left(c_{2}+1\right) \sigma_{1}^{2} S_{0}^{2}-\sigma_{3}^{2} V_{0}^{2}\right\} d t \\
+\sigma_{1}\left(x+S_{0}\right)\left[\left(c_{2}+1\right)(x+y)+z\right] d B_{1}(t) \\
+\sigma_{2} y\left[c_{1}+\left(c_{2}+1\right)(x+y)+z\right] d B_{2}(t) \\
+\sigma_{3}(x+y+z)\left(z+V_{0}\right) d B_{3}(t) .
\end{aligned}
$$

Integrating this from 0 to $t$ and taking the expectation, we have

$$
\begin{aligned}
& E W(t)-W(0) \\
& \leqslant-E \int_{0}^{t}\left\{\left[\mu\left(\frac{2(\mu+p)}{\varepsilon}+1\right)-\left(c_{2}+1\right) \sigma_{1}^{2}\right] x^{2}(r)\right. \\
& +\left[(\mu+\alpha)\left(1+\frac{2 \mu}{\varepsilon}\right)-\frac{\alpha}{4 \rho}\right. \\
& \left.-\left(c_{2}+1\right) \sigma_{2}^{2}\right] y^{2}(r) \\
& +\left[(\mu-\rho \alpha)-\sigma_{3}^{2}\right] z^{2}(r) \\
& \left.-\left(c_{2}+1\right) \sigma_{1}^{2} S_{0}^{2}-\sigma_{3}^{2} V_{0}^{2}\right\} d r
\end{aligned}
$$

Hence,

$$
\begin{gathered}
\limsup _{t \rightarrow \infty} \frac{1}{t} E \int_{0}^{t}\left\{\left[\mu\left(\frac{2(\mu+p)}{\varepsilon}+1\right)\right.\right. \\
\left.-\left(c_{2}+1\right) \sigma_{1}^{2}\right] x^{2}(r) \\
+\left[(\mu+\alpha)\left(1+\frac{2 \mu}{\varepsilon}\right)-\frac{\alpha}{4 \rho}\right. \\
\left.\quad-\left(c_{2}+1\right) \sigma_{2}^{2}-\frac{\alpha}{4 \rho}\right] y^{2}(r) \\
\left.+\left[(\mu-\rho \alpha)-\sigma_{3}^{2}\right] z^{2}(r)\right\} d r \\
\leqslant\left(c_{2}+1\right) \sigma_{1}^{2} S_{0}^{2}+\sigma_{3}^{2} V_{0}^{2} .
\end{gathered}
$$

Consequently,

$$
\begin{aligned}
\limsup _{t \rightarrow \infty} \frac{1}{t} E \int_{0}^{t}\{[ & \mu\left(\frac{2(\mu+p)}{\varepsilon}+1\right) \\
& \left.-\left(c_{2}+1\right) \sigma_{1}^{2}\right] \\
& \times\left(S(r)-S_{0}\right)^{2} \\
+ & {\left[(\mu+\alpha)\left(1+\frac{2 \mu}{\varepsilon}\right)\right.} \\
& -\left[(\mu-\rho \alpha)-\sigma_{3}^{2}\right] \\
& \left.\times\left(V(r)-V_{0}\right)^{2}\right\} d r \\
& \\
\leqslant\left(c_{2}+1\right) \sigma_{1}^{2} S_{0}^{2}+ & \sigma_{3}^{2} V_{0}^{2} .
\end{aligned}
$$

This finishes the proof of Theorem 2 . 
Remark 3. From Theorem 2, we show the solution of system (6) will oscillate around the disease-free equilibrium of system (1) under some conditions and the disturbance intensity is proportional to the intensity of the white noise. In a biological view, as the intensity of stochastic perturbations is small, we consider the disease will die out.

Besides, if $\sigma_{1}=0$ and $\sigma_{3}=0$, then $P_{0}$ is also the diseasefree equilibrium of system (6). From the proof of Theorem 2, we get

$$
\begin{gathered}
L W \leqslant-\left\{\mu\left[\frac{2(\mu+p)}{\varepsilon}+1\right]\right. \\
\left.-\left(c_{2}+1\right) \sigma_{1}^{2}\right\} x^{2} \\
-\left[(\mu+\alpha)\left(1+\frac{2 \mu}{\varepsilon}\right)\right. \\
\left.-\frac{\alpha}{4 \rho}-\left(c_{2}+1\right) \sigma_{2}^{2}\right] y^{2} \\
-\left[(\mu-\rho \alpha)-\sigma_{3}^{2}\right] z^{2} .
\end{gathered}
$$

Thus, the solution of system (6) is stochastically asymptotically stable in the large if $(\mu+2)(1+2 \mu / \varepsilon)-\alpha / 4 \rho>\left(c_{2}+1\right) \sigma_{2}^{2}$.

\section{Ergodicity of System (6)}

In studying epidemic dynamical system, we are also interested in when the disease will prevail and persist in a population. In the deterministic models, the problem is solved by showing that the endemic equilibrium of corresponding model is a global attractor or is globally asymptotically stable. But there is no endemic equilibrium in system (6). In this section, based on the theory of Has'minskil [26] (see the appendix), we show that there is a stationary distribution subjected to some conditions on $R_{0}$ and the parameters of the model which reveals that the disease will prevail also.

System (6) can be written as a form of system (A.8) (see the appendix).

Consider

$$
\begin{aligned}
& d\left(\begin{array}{c}
S \\
I \\
V
\end{array}\right) \\
& =\left(\begin{array}{c}
(1-q) A-\beta S(t) I(t)-(\mu+p) S(t)+\gamma I(t)+\varepsilon V(t) \\
\beta S(t) I(t)-(\mu+\gamma+a) I(t) \\
q A+p S(t)-(\mu+\varepsilon) V(t)
\end{array}\right) d t \\
& +\left(\begin{array}{c}
\sigma_{1} S \\
0 \\
0
\end{array}\right) d B_{1}(t)+\left(\begin{array}{c}
0 \\
\sigma_{2} I \\
0
\end{array}\right) d B_{2}(t) \\
& +\left(\begin{array}{c}
0 \\
0 \\
\sigma_{3} V
\end{array}\right) d B_{3}(t),
\end{aligned}
$$

and the diffusion matrix is $A=\operatorname{diag}\left(\sigma_{1}^{2} S^{2}, \sigma_{2}^{2} I^{2}, \sigma_{3}^{2} V^{2}\right)$.
Remark 4. Theorem 1 shows that there exists a unique positive solution $Y(t)=(S(t), I(t), V(t))$ of system (6). Besides, from the proof of Theorem 1, we obtain $L V \leqslant K$. Define $\widetilde{V}=V+K$, then $L \widetilde{V} \leq \widetilde{V}$, and it is clear that $\widetilde{V}_{k}=$ $\inf _{X \in R_{+}^{3} \backslash C_{k}} \widetilde{V}(Y) \rightarrow \infty$ as $k \rightarrow \infty$, where $C_{k}=(1 / k, k) \times$ $(1 / k, k) \times(1 / k, k)$. Hence, by Remark 2 of Theorem 4.1 of Has'minskii (1980, page 86) [26], we obtain that the solution $Y(t)$ is a homogeneous Markov process in $R_{+}^{3}$.

Theorem 5. If $R_{0}>1, \alpha^{2}<4 \mu(\mu+\alpha)(1+2 \mu / \varepsilon)$, and

$$
\begin{gathered}
0<\sigma_{1}^{2}<\frac{\mu}{c_{2}+1}\left(\frac{2(\mu+p)}{\varepsilon}+1\right), \\
0<\sigma_{2}^{2}<\frac{1}{c_{2}+1}\left[(\mu+\alpha)\left(1+\frac{2 \mu}{\varepsilon}\right)-\frac{\alpha}{4 \rho}\right], \\
0<\sigma_{3}^{2}<\mu-\rho \alpha,
\end{gathered}
$$

such that

$$
\begin{gathered}
\delta<\min \left\{\left[\mu\left(\frac{2(\mu+p)}{\varepsilon}+1\right)\right.\right. \\
\left.-\left(c_{2}+1\right) \sigma_{1}^{2}\right]\left(S^{*}\right)^{2}, \\
\quad\left[(\mu+\alpha)\left(1+\frac{2 \mu}{\varepsilon}\right)-\frac{\alpha}{4 \rho}\right. \\
\left.-\left(c_{2}+1\right) \sigma_{2}^{2}\right]\left(I^{*}\right)^{2}, \\
\left.\left(\mu-\rho \alpha-\sigma_{3}^{2}\right)\left(V^{*}\right)^{2}\right\},
\end{gathered}
$$

then, for any initial value $Y(0) \in R_{+}^{3}$, there is a stationary distribution $\mu(\cdot)$ for system (6) and it has an ergodic property, where $\delta=\sigma_{2}^{2} I^{*} / 2+\left(c_{2}+1\right) \sigma_{1}^{2}\left(S^{*}\right)^{2}+\left(c_{2}+1\right) \sigma_{2}^{2}\left(I^{*}\right)^{2}+\sigma_{3}^{2}\left(V^{*}\right)^{2}$, $P^{*}=\left(S^{*}, I^{*}, V^{*}\right)$ is the endemic proportion equilibrium of system (1), and $c_{2}$ and $\rho$ are the same constants as in Theorem 2.

Proof. If $R_{0}>1$, there is an endemic proportion equilibrium $P^{*}=\left(S^{*}, I^{*}, V^{*}\right)$ of system $(1)$; then

$$
\begin{gathered}
(1-q) A=\beta S^{*} I^{*}+(\mu+p) S^{*}-\gamma I^{*}-\varepsilon V^{*}, \\
\beta S^{*} I^{*}=(\mu+\gamma+\alpha) I^{*}, \\
q A+p S^{*}=(\mu+\varepsilon) V^{*} .
\end{gathered}
$$

Define a $C^{2}$-function $W: \mathbb{R}_{+}^{3} \rightarrow \mathbb{R}_{+}$by

$W(S, I, V)$

$$
=c_{1}\left(I-I^{*}-I^{*} \log \frac{I}{I^{*}}\right)
$$




$$
\begin{aligned}
& +\frac{c_{2}}{2}\left[\left(S-S^{*}\right)+\left(I-I^{*}\right)\right]^{2} \\
& +\frac{1}{2}\left[\left(S-S^{*}\right)+\left(I-I^{*}\right)+\left(V-V^{*}\right)\right]^{2} \\
:= & c_{1} W_{1}+c_{2} W_{2}+W_{3},
\end{aligned}
$$

where $c_{1}=1 / \beta[2 \mu(2 \mu+p+\alpha) / \varepsilon+(2 \mu+\alpha)]$. The nonnegativity of the function can be observed from $u-1-\log u \geq 0$ on $u>0$. Let $L$ be the generating operator of system (6). Then we get

$$
\begin{aligned}
& L W_{1}=\beta\left(S-S^{*}\right)\left(I-I^{*}\right)+\frac{1}{2} \sigma_{2}^{2} I^{*}, \\
L W_{2}= & -(\mu+p)\left(S-S^{*}\right)^{2}-(\mu+\alpha)\left(I-I^{*}\right)^{2} \\
& -[(\mu+p)+(\mu+\alpha)]\left(S-S^{*}\right)\left(I-I^{*}\right) \\
& +\varepsilon\left(S-S^{*}\right)\left(V-V^{*}\right)+\varepsilon\left(I-I^{*}\right)\left(V-V^{*}\right) \\
& +\frac{1}{2}\left(\sigma_{1}^{2} S^{2}+\sigma_{2}^{2} I^{2}\right), \\
L W_{3}= & -\mu\left(S-S^{*}\right)^{2}-(\mu+\alpha)\left(I-I^{*}\right)^{2} \\
& -\mu\left(V-V^{*}\right)^{2}-(2 \mu+\alpha)\left(S-S^{*}\right)\left(I-I^{*}\right) \\
& -2 \mu\left(S-S^{*}\right)\left(V-V^{*}\right) \\
& -(2 \mu+\alpha)\left(I-I^{*}\right)\left(V-V^{*}\right) \\
& +\frac{1}{2}\left(\sigma_{1}^{2} S^{2}+\sigma_{2}^{2} I^{2}+\sigma_{3}^{2} V^{2}\right) .
\end{aligned}
$$

Then

$$
\begin{aligned}
L W= & c_{1} L W_{1}+c_{2} L W_{2}+L W_{3} \\
= & {\left[c_{1} \beta-c_{2}(2 \mu+p+\alpha)-(2 \mu+\alpha)\right] } \\
& \times\left(S-S^{*}\right)\left(I-I^{*}\right) \\
& -\left[c_{2}(\mu+p)+\mu\right]\left(S-S^{*}\right)^{2} \\
& -\left(c_{2}+1\right)(\mu+\alpha)\left(I-I^{*}\right)^{2} \\
& -\mu\left(V-V^{*}\right)^{2}+\left(c_{2} \varepsilon-2 \mu\right)\left(S-S^{*}\right)\left(V-V^{*}\right) \\
& +\left[c_{2} \varepsilon-(2 \mu+\alpha)\right]\left(I-I^{*}\right)\left(V-V^{*}\right) \\
& +\frac{1}{2} \sigma_{2}^{2} I^{*}+\frac{c_{2}}{2}\left(\sigma_{1}^{2} S^{2}+\sigma_{2}^{2} I^{2}\right) \\
& +\frac{1}{2}\left(\sigma_{1}^{2} S^{2}+\sigma_{2}^{2} I^{2}+\sigma_{3}^{2} V^{2}\right) .
\end{aligned}
$$

Note that

$$
c_{1} \beta-c_{2}(2 \mu+p+\alpha)-(2 \mu+\alpha)=0, \quad c_{2} \varepsilon-2 \mu=0 .
$$

Then

$$
\begin{aligned}
L W= & -\mu\left[\frac{2(\mu+p)}{\varepsilon}+1\right]\left(S-S^{*}\right)^{2} \\
& -\left(1+\frac{2 \mu}{\varepsilon}\right)(\mu+\alpha)\left(I-I^{*}\right)^{2} \\
& -\mu\left(V-V^{*}\right)^{2}-\alpha\left(I-I^{*}\right)\left(V-V^{*}\right) \\
& +\frac{1}{2} \sigma_{2}^{2} I^{*}+\frac{c_{2}+1}{2} \sigma_{1}^{2} S^{2} \\
+ & \frac{c_{2}+1}{2} \sigma_{2}^{2} I^{2}+\sigma_{3}^{2} V^{2} \\
\leqslant & -\left[\mu\left(\frac{2(\mu+p)}{\varepsilon}+1\right)-\left(c_{2}+1\right) \sigma_{1}^{2}\right]\left(S-S^{*}\right)^{2} \\
& -\left[(\mu+\alpha)\left(1+\frac{2 \mu}{\varepsilon}\right)\right. \\
& \left.-\frac{\alpha}{4 \rho}-\left(c_{2}+1\right) \sigma_{2}^{2}\right]\left(I-I^{*}\right)^{2} \\
& -\left(\mu-\rho \alpha-\sigma_{3}^{2}\right)\left(V-V^{*}\right)^{2} \\
& +\frac{1}{2} \sigma_{2}^{2} I^{*}+\left(c_{2}+1\right) \sigma_{1}^{2}\left(S^{*}\right)^{2} \\
+ & \left(c_{2}+1\right) \sigma_{2}^{2}\left(I^{*}\right)^{2}+\sigma_{3}^{2}\left(V^{*}\right)^{2} \\
& -\left[\mu\left(\frac{2(\mu+p)}{\varepsilon}+1\right)-\left(c_{2}+1\right) \sigma_{1}^{2}\right]\left(S-S^{*}\right)^{2} \\
& -\left[\left(c_{2}+1\right) \sigma_{2}^{2}\right]\left(I-I^{*}\right)^{2} \\
& {\left[1+\frac{2 \mu}{\varepsilon}\right) } \\
& \left.-\sigma_{3}^{2}\right)\left(V-V^{*}\right)^{2}+\delta \\
\hline &
\end{aligned}
$$

where Young's inequality is used and

$$
\delta=\frac{1}{2} \sigma_{2}^{2} I^{*}+\left(c_{2}+1\right) \sigma_{1}^{2}\left(S^{*}\right)^{2}+\left(c_{2}+1\right) \sigma_{2}^{2}\left(I^{*}\right)^{2}+\sigma_{3}^{2}\left(V^{*}\right)^{2} .
$$

If $\alpha^{2}<4 \mu(\mu+\alpha)(1+2 \mu / \varepsilon)$, choose $\rho$ such that

$$
(\mu+\alpha)\left(1+\frac{2 \mu}{\varepsilon}\right)-\frac{\alpha}{4 \rho}>0, \quad \mu-\rho \alpha>0 .
$$


Note that

$$
\begin{aligned}
\delta<\min \left\{\left[\mu\left(\frac{2(\mu+p)}{\varepsilon}+1\right)\right.\right. \\
\left.-\left(c_{2}+1\right) \sigma_{1}^{2}\right]\left(S^{*}\right)^{2}, \\
\quad\left[(\mu+\alpha)\left(1+\frac{2 \mu}{\varepsilon}\right)-\frac{\alpha}{4 \rho}\right. \\
\left.-\left(c_{2}+1\right) \sigma_{2}^{2}\right]\left(I^{*}\right)^{2}, \\
\\
{\left.\left[\left(\mu-\rho \alpha-\sigma_{3}^{2}\right)\right]\left(V^{*}\right)^{2}\right\} . }
\end{aligned}
$$

Thus the ellipsoid

$$
\begin{gathered}
-\left[\mu\left(\frac{2(\mu+p)}{\varepsilon}+1\right)-\left(c_{2}+1\right) \sigma_{1}^{2}\right]\left(S-S^{*}\right)^{2} \\
-\left[(\mu+\alpha)\left(1+\frac{2 \mu}{\varepsilon}\right)\right. \\
\left.-\frac{\alpha}{4 \rho}-\left(c_{2}+1\right) \sigma_{2}^{2}\right]\left(I-I^{*}\right)^{2} \\
-\left(\mu-\rho \alpha-\sigma_{3}^{2}\right)\left(V-V^{*}\right)^{2}+\delta=0
\end{gathered}
$$

lies entirely in $R_{+}^{3}$. We can take $U$ to be a neighborhood of the ellipsoid with $\bar{U} \subseteq E_{l}=R_{+}^{3}$, so, for $x \in R_{+}^{3} \backslash U$, $L V \leq-M$ ( $M$ is a positive constant), which implies that condition (B.2) in Lemma A.2 is satisfied. Hence, the solution $Y(t)=(S(t), I(t), V(t))$ is recurrent in the domain $U$, which, together with Lemma A.4 and Remark 4, implies that $Y(t)=$ $(S(t), I(t), V(t))$ is recurrent in any bounded domain $D \subset R_{+}^{3}$. Besides, for all $D$, there is

$$
M=\min \left\{\sigma_{1}^{2} S^{2}, \sigma_{2}^{2} I^{2}, \sigma_{3}^{2} V^{2}\right\}>0,
$$

such that

$$
\sum_{i, j=1}^{3} a_{i j} \xi_{i} \xi_{j}=\sigma_{1}^{2} S^{2} \xi_{1}^{2}+\sigma_{2}^{2} I^{2} \xi_{2}^{2}+\sigma_{3}^{2} V^{2} \xi_{3}^{2} \geq M\|\xi\|^{2}
$$

for all $Y \in \bar{D}, \xi \in R^{3}$, which implies that condition (B.1) is also satisfied. Therefore, the stochastic system (6) has a stationary distribution $\mu(\cdot)$, and it is ergodic.

\section{Numerical Simulations}

In order to confirm the results above, we numerically simulate the solution of system (6) with given initial value and the parameters. Using Milstein's higher order method mentioned in [27], we get the discretization equation of system (6):

$$
\begin{aligned}
S_{k+1}= & S_{k}+\left((1-q) A-\beta S_{k} I_{k}\right. \\
& \left.-(\mu+p) S_{k}+\gamma I_{k}+\varepsilon V_{k}\right) \Delta t \\
& +\sigma_{1} S_{k} \sqrt{\Delta t} \xi_{1, k}+\frac{\sigma_{1}^{2}}{2} S_{k} \Delta t\left(\xi_{1, k}^{2}-1\right), \\
I_{k+1}= & I_{k}+\left(\beta S_{k} I_{k}-(\mu+\gamma+\alpha) I_{k}\right) \Delta t+\sigma_{2} I_{k} \sqrt{\Delta t} \xi_{2, k} \\
& +\frac{\sigma_{2}^{2}}{2} I_{k} \Delta t\left(\xi_{2, k}^{2}-1\right), \\
V_{k+1}= & V_{k}+\left(q A+p S_{k}-(\mu+\varepsilon) V_{k}\right) \Delta t+\sigma_{3} V_{k} \sqrt{\Delta t} \xi_{3, k} \\
& +\frac{\sigma_{3}^{2}}{2} V_{k} \Delta t\left(\xi_{3, k}^{2}-1\right),
\end{aligned}
$$

where $\xi_{1, k}, \xi_{2, k}$, and $\xi_{3, k}, k=1,2, \ldots, n$, are the independent Gaussian random variables $N(0,1)$. Choosing suitable parameters in the system, by Matlab we get the simulation figures with initial value $(S(0), I(0), V(0))=(0.8,0.4,0.5)$ and time step $\Delta t=0.001$.

Example 6. Throughout the paper we will assume that the unit of time is one day, and the population sizes are measured in units of 1 million. Choose the parameters in system (6) as follows: $A=0.2, q=0.5, \beta=0.5, \mu=0.2, p=0.3, \gamma=0.2$, $\varepsilon=0.1, \alpha=0.3, \sigma_{1}=0.05, \sigma_{2}=0.1$, and $\sigma_{3}=0.05$. Note that

$$
\begin{gathered}
R_{0}=\frac{A \beta(\mu(1-q)+\varepsilon)}{\mu(\mu+\gamma+\alpha)(\mu+\varepsilon+p)}=0.4<1, \\
\alpha^{2}=0.09<4 \mu(\mu+\alpha)\left(1+\frac{2 \mu}{\varepsilon}\right)=2,
\end{gathered}
$$

and $\sigma_{1}, \sigma_{2}$, and $\sigma_{3}$ satisfy condition (14), then by Theorem 2 , we show the solution of system (6) will oscillate around the disease-free equilibrium $P_{0}\left(S_{0}, I_{0}, V_{0}\right)$ of system (1) in time which is globally asymptotically stable. Besides Theorem 2 tells us that the difference between the perturbed solution and $P_{0}$ is only related with white noises $\sigma_{1}$ and $\sigma_{3}$. Numerical simulations are shown in Figure 1.

Example 7. In Figures 2 and 3, we choose the parameters in system (6) as follows: $A=0.9, q=0.1, \beta=0.8$, $\mu=0.4, p=0.1, \gamma=0.1, \varepsilon=0.2, \alpha=0.1, \sigma_{1}=0.1$, $\sigma_{2}=0.1$, and $\sigma_{3}=0.1$. Note that $R_{0}=2.486>1$ and $\alpha^{2}<4 \mu(\mu+\alpha)(1+2 \mu / \varepsilon)=4$; then the equilibrium $P^{*}\left(S^{*}, I^{*}, V^{*}\right)$ of system (1) is globally asymptotically stable. But the white noise may make system (1) appear as different phenomena. In detail, the conditions (30) and (31) are also satisfied. Therefore, as Theorem 5 stated, there is a stationary distribution (see the histograms in the middle in Figure 2). In addition, the left pictures in Figure 2 show that the solution of system (6) has fluctuating in a small neighborhood. Moreover, from Figure 3, we find that 95\% or more of the population distribution lies within a neighborhood, which 




(a)

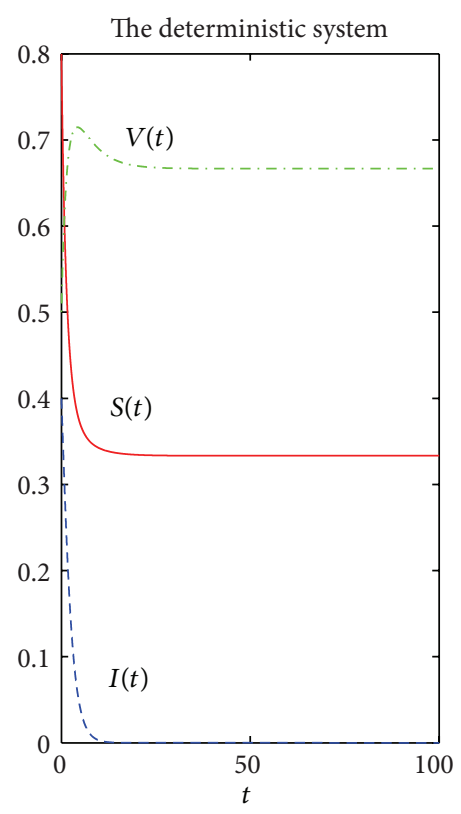

(b)

FIGURE 1: Computer simulation of the paths $S(t), I(t)$, and $V(t)$ for the SDE SIS model (6) with vaccination and its corresponding deterministic model (1) for parameter values $A=0.2, q=0.5, \beta=0.5, \mu=0.2, p=0.3, \gamma=0.2, \varepsilon=0.1, \alpha=0.3, \sigma_{1}=0.05, \sigma_{2}=0.1$, and $\sigma_{3}=0.05$.

can be imagined a circular or elliptic region centered at $P^{*}\left(S^{*}, I^{*}, V^{*}\right)$ (see the red point in Figure 3). All of these imply system (6) has stochastic stability.

Testing these data for normality, all tests used were highly significant, conclusively rejecting normality in all cases. This is not surprising in view of the very large sample sizes $(10,000)$, as even moderate deviations from the tested distribution will be significant; however the normal quantilequantile plots (see the QQ plots in the right in Figure 2) suggest that these data are not far from being normally distributed for smaller values of $\sigma_{i}(i=1,2,3)$.

\section{Conclusions}

As most real world problems are not deterministic, incorporating stochastic effects into the model give us a more realistic way of modeling epidemic models. In this paper, we have considered stochastic SIS epidemic models with vaccination. We first proved the positivity of the solutions. When the perturbation and the disease-related death rate are small, we illustrated the dynamical behavior of the SDE model according to $R_{0} \leq 1$ or $R_{0}>1$. When $R_{0} \leq 1$, we proved that the asymptotic behavior around the disease-free equilibrium of the deterministic model. When $R_{0}>1$, we also proved that the SDE model has the ergodic property as the fluctuation is small, where the positive solution converges weakly to the unique stationary distribution. Simulations are also carried out to verify our analytical results.

\section{Appendix}

Here, we give some basic theory in stochastic differential equations (see [18]).
Throughout this paper, unless otherwise specified, let $\left(\Omega,\left\{F_{t}\right\}_{t>0}, P\right)$ be a complete probability space with a filtration $\left\{F_{t}\right\}_{t \geq 0}$ satisfying the usual conditions (i.e., it is right continuous, and $F_{0}$ contains all P-null sets). Denote

$$
\begin{aligned}
& \mathbb{R}_{+}^{d}=\left\{x \in \mathbb{R}^{d}: x_{i}>0 \quad \forall 1 \leqslant i \leqslant d\right\}, \\
& \overline{\mathbb{R}}_{+}^{d}=\left\{x \in \mathbb{R}^{d}: x_{i} \geq 0 \quad \forall 1 \leqslant i \leqslant d\right\} .
\end{aligned}
$$

In general, consider $n$-dimensional stochastic differential equation:

$$
d x(t)=f(x(t), t) d t+g(x(t), t) d B(t), \quad \text { for } t \geq t_{0},
$$

with initial value $x\left(t_{0}\right)=x_{0} \in R^{d} . B(t)$ denotes $n$ dimensional standard Brownian motion defined on the above probability space. Define the differential operator $L$ associated with (A.2) by

$$
\begin{aligned}
L= & \frac{\partial}{\partial t}+\sum_{i=1}^{d} f_{i}(x, t) \frac{\partial}{\partial x_{i}} \\
& +\frac{1}{2} \sum_{i, j=1}^{d}\left[g^{T}(x, t) g(x, t)\right]_{i j} \frac{\partial^{2}}{\partial x_{i} \partial x_{j}} .
\end{aligned}
$$

If $L$ acts on a function $V \in C^{2,1}\left(\mathbb{R}^{d} \times \overline{\mathbb{R}}_{+} ; \overline{\mathbb{R}}_{+}\right)$, then

$$
\begin{aligned}
L V(x, t)= & V_{t}(x, t)+V_{x}(x, t) f(x, t) \\
& +\frac{1}{2} \operatorname{trace}\left[g^{T}(x, t) V_{x x}(x, t) g(x, t)\right],
\end{aligned}
$$






(a)

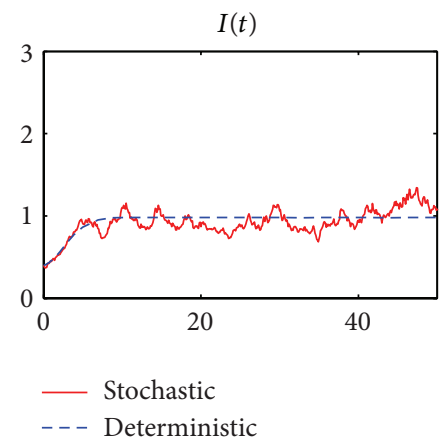

(d)

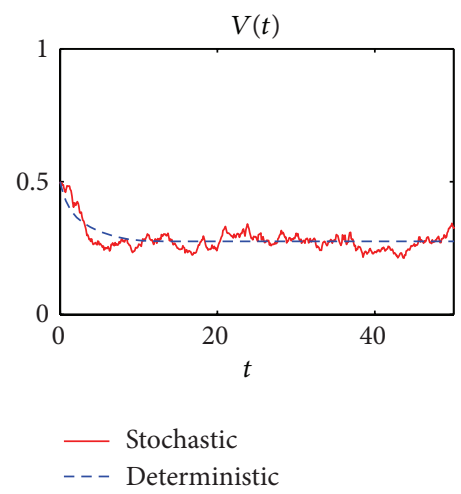

(g)

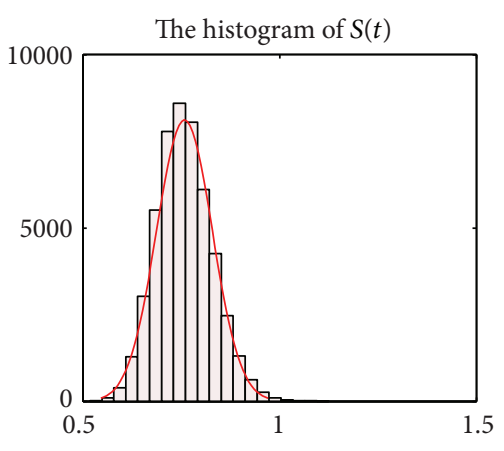

(b)

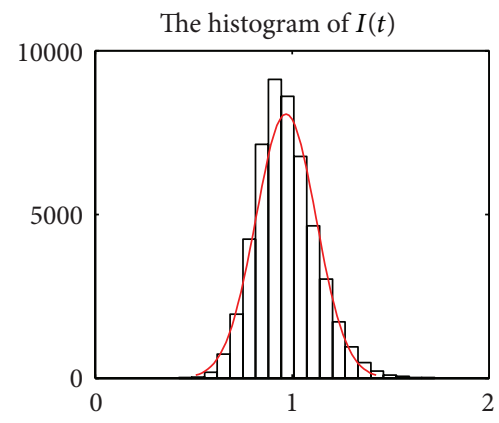

(e)

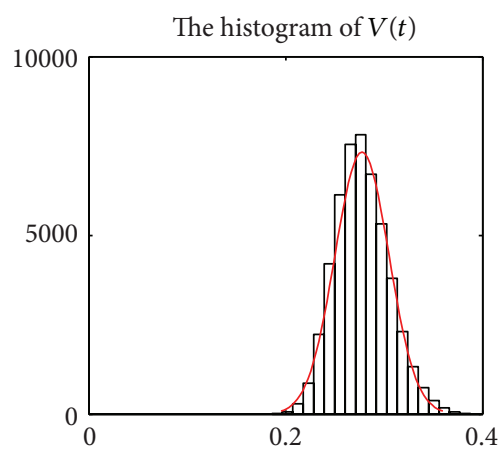

(h)

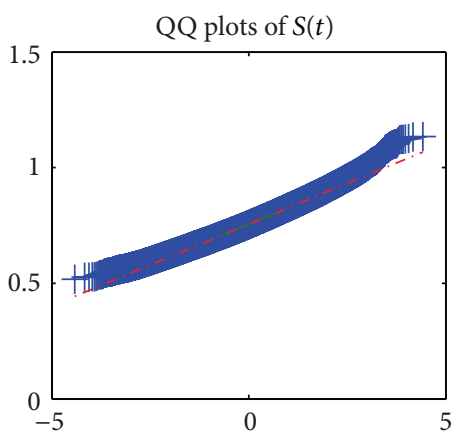

(c)

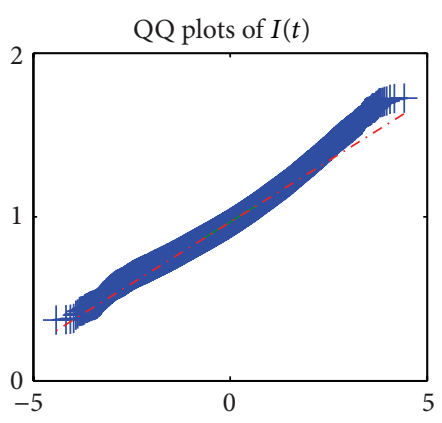

(f)

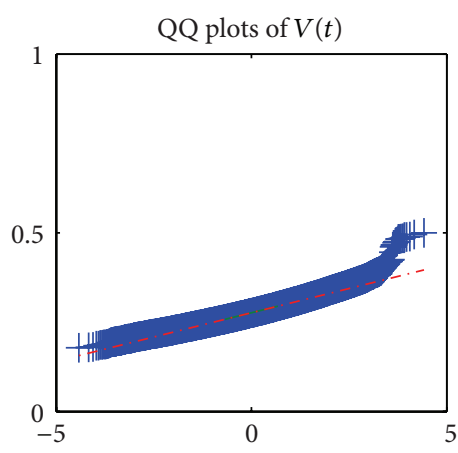

(i)

Figure 2: The solution of the stochastic system (6), its histogram, and normal quantile-quantile plots for parameter values $A=0.9, q=0.1$, $\beta=0.8, \mu=0.4, p=0.1, \gamma=0.1, \varepsilon=0.2, \alpha=0.1, \sigma_{1}=0.1, \sigma_{2}=0.1$, and $\sigma_{3}=0.1$.

where $V_{t}=\partial V / \partial t, V_{x}=\left(\partial V / \partial x_{1}, \ldots, \partial V / \partial x_{d}\right)$, and $V_{x x}=$ $\left(\partial^{2} V / \partial x_{i} \partial x_{j}\right)_{d \times d}$. By Itô's formula, if $x(t) \in R^{d}$, then

$$
\begin{aligned}
d V(x(t), t)= & L V(x(t), t) d t \\
& +V_{x}(x(t), t) g(x(t), t) d B(t) .
\end{aligned}
$$

Consider (A.2) assume $f(0, t)=0$ and $g(0, t)=0$ for all $t \geq$ $t_{0}$. So $x(t) \equiv 0$ is a solution of (A.2) called the trivial solution or equilibrium position.
Lemma A.1 (strong law of large numbers). Let $M=\left\{M_{t}\right\}_{t \geq 0}$ be a real-value continuous local martingale vanishing at $t=0$. Then

$$
\lim _{t \rightarrow \infty}\langle M, M\rangle_{t}=\infty \quad \text { a.s. } \Longrightarrow \lim _{t \rightarrow \infty} \frac{M_{t}}{\langle M, M\rangle_{t}}=0 \text {. a.s. }
$$

and also

$$
\limsup _{t \rightarrow \infty} \frac{\langle M, M\rangle_{t}}{t}<\infty \quad \text { a.s. } \Longrightarrow \lim _{t \rightarrow \infty} \frac{M_{t}}{t}=0 \text {. a.s. }
$$




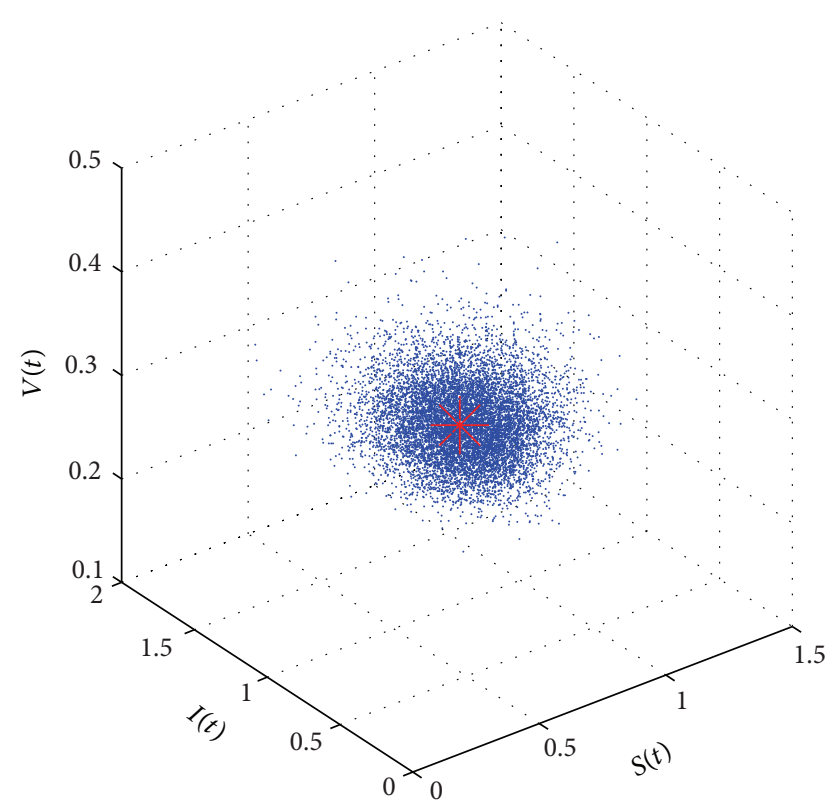

FIgURe 3: Population distribution around point $P^{*}\left(S^{*}, I^{*}, V^{*}\right)=$ $(0.75,0.98,0.275)$ (red point) corresponding to Figure 2 .

Next, there is some theory about stationary distributions (see Has'minskiur [26], 1980).

Let $X(t)$ be a homogeneous Markov process in $E_{l}\left(E_{l}\right.$ denotes Euclidean $l$-space) described by

$$
d X(t)=b(X) d t+\sum_{r=1}^{k} g_{r}(X) d B_{r}(t)
$$

The diffusion matrix is $A(x)=\left(a_{i j}(x)\right), a_{i j}(x)=$ $\sum_{r=1}^{k} g_{r}^{i}(x) g_{r}^{j}(x)$.

Assumption B. There exists a bounded domain $U \subset E_{l}$ with regular boundary $\Gamma$, having the following properties.

(B.1) In the domain $U$ and some neighborhood thereof, the smallest eigenvalue of the diffusion matrix $A(x)$ is bounded away from zero.

(B.2) If $x \in E_{l} \backslash U$, the mean time $\tau$ at which a path issuing from $x$ reaches the set $U$ is finite and $\sup _{x \in K} E_{x} \tau<\infty$ for every compact subset $K \subset E_{l}$.

Lemma A.2 (see [26]). If (B) holds, then the Markov process $X(t)$ has a stationary distribution $\mu(\cdot)$. Let $f(\cdot)$ be a function integrable with respect to the measure $\mu$. Then

$$
\begin{array}{r}
P_{x}\left\{\lim _{T \rightarrow \infty} \frac{1}{T} \int_{0}^{T} f(X(t)) d t=\int_{E_{l}} f(x) \mu(d x)\right\}=1, \\
\forall x \in E_{l} .
\end{array}
$$

Remark A.3. The proof is given in Has'minskil [26]. The existence of a stationary distribution with a density is given in Theorem 4.1, page 119, and Lemma 9.4, page 138. The weak convergence and the ergodicity are obtained in Theorem 5.1, page 121, and Theorem 7.1, page 130 .
To validate (B.1), it suffices to prove that $F$ is uniformly elliptical in any bounded domain $D$, where $F u=b(x) \cdot u_{x}+$ $(1 / 2) \operatorname{tr}\left(A(x) u_{x x}\right)$; that is, there is a positive number $M$ such that $\sum_{i, j=1}^{k} a_{i j}(x) \xi_{i} \xi_{j} \geq M|\xi|^{2}, x \in \bar{D}, \xi \in R^{k}$ (see chapter 3, page 103 of Gard [28] and Rayleigh's principle in Strang [29], chapter 6, page 349). To verify (B.2), it is sufficient to show that there exist some neighborhood $U$ and a nonnegative $C_{2}-$ function such that, for any $E_{l} \backslash U, L V$ is negative (for details refer to Zhu and Yin [30], page 1163).

Lemma A.4. Let $X(t)$ be a regular temporally homogeneous Markov process in $E_{l}$. If $X(t)$ is recurrent relative to some bounded domain $U$, then it is recurrent relative to any nonempty domain in $E_{l}$.

\section{Acknowledgments}

The work was supported by Program for Changjiang Scholars and Innovative Research Team in University, NSFC of China (no. 10971021), the Ph.D. Programs Foundation of Ministry of China (no. 200918), and the Scientific and Technological Research Project of Jilin Province's Education Department (no. 2012244).

\section{References}

[1] F. H. Chen, "A susceptible-infected epidemic model with voluntary vaccinations," Journal of Mathematical Biology, vol. 53, no. 2, pp. 253-272, 2006.

[2] E. Shim, Z. Feng, M. Martcheva, and C. Castillo-Chavez, "An age-structured epidemic model of rotavirus with vaccination," Journal of Mathematical Biology, vol. 53, no. 4, pp. 719-746, 2006.

[3] I. Moneim and D. Greenhalgh, "Threshold and stability results for an sirs epidemic model with ageneral periodic vaccination strategy," Journal of Biological Systems, vol. 13, pp. 131-150, 2005.

[4] D. Greenhalgh, "Hopf bifurcation in epidemic models with a latent period and nonpermanent immunity," Mathematical and Computer Modelling, vol. 25, no. 2, pp. 85-107, 1997.

[5] D. Greenhalgh, "Some results for an SEIR epidemic model with density dependence in the death rate," IMA Journal of Mathematics Applied in Medicine and Biology, vol. 9, no. 2, pp. 67-106, 1992.

[6] J. Li and Z. Ma, "Qualitative analyses of SIS epidemic model with vaccination and varying total population size," Mathematical and Computer Modelling, vol. 35, no. 11-12, pp. 1235-1243, 2002.

[7] J. Li and Z. Ma, "Global analysis of SIS epidemic models with variable total population size," Mathematical and Computer Modelling, vol. 39, no. 11-12, pp. 1231-1242, 2004.

[8] L. Arnold, W. Horsthemke, and J. W. Stucki, "The influence of external real and white noise on the Lotka-Volterra model," Biometrical Journal, vol. 21, no. 5, pp. 451-471, 1979.

[9] A. Bahar and X. Mao, "Stochastic delay Lotka-Volterra model," Journal of Mathematical Analysis and Applications, vol. 292, no. 2, pp. 364-380, 2004.

[10] T. C. Gard, "Persistence in stochastic food web models," Bulletin of Mathematical Biology, vol. 46, no. 3, pp. 357-370, 1984.

[11] L. Imhof and S. Walcher, "Exclusion and persistence in deterministic and stochastic chemostat models," Journal of Differential Equations, vol. 217, no. 1, pp. 26-53, 2005. 
[12] C. Ji and D. Jiang, "Dynamics of a stochastic density dependent predator-prey system with Beddington-DeAngelis functional response," Journal of Mathematical Analysis and Applications, vol. 381, no. 1, pp. 441-453, 2011.

[13] C. Ji, D. Jiang, and N. Shi, "Multigroup SIR epidemic model with stochastic perturbation," Physica A, vol. 390, pp. 1747-1762, 2011.

[14] D. Jiang, C. Ji, N. Shi, and J. Yu, "The long time behavior of DI SIR epidemic model with stochastic perturbation," Journal of Mathematical Analysis and Applications, vol. 372, no. 1, pp. 162-180, 2010.

[15] R. Z. Khasminskii and F. C. Klebaner, "Long term behavior of solutions of the Lotka-Volterra system under small random perturbations," The Annals of Applied Probability, vol. 11, no. 3, pp. 952-963, 2001.

[16] X. Mao, S. Sabanis, and E. Renshaw, "Asymptotic behaviour of the stochastic Lotka-Volterra model," Journal of Mathematical Analysis and Applications, vol. 287, no. 1, pp. 141-156, 2003.

[17] X. Mao, G. Marion, and E. Renshaw, "Environmental Brownian noise suppresses explosions in population dynamics," Stochastic Processes and their Applications, vol. 97, no. 1, pp. 95-110, 2002.

[18] X. Mao, Stochastic Differential Equations and Applications, Horwood, Chichester, UK, 1997.

[19] M. Carletti, "On the stability properties of a stochastic model for phage-bacteria interaction in open marine environment," Mathematical Biosciences, vol. 175, no. 2, pp. 117-131, 2002.

[20] N. Dalal, D. Greenhalgh, and X. Mao, "A stochastic model of AIDS and condom use," Journal of Mathematical Analysis and Applications, vol. 325, no. 1, pp. 36-53, 2007.

[21] N. Dalal, D. Greenhalgh, and X. Mao, "A stochastic model for internal HIV dynamics," Journal of Mathematical Analysis and Applications, vol. 341, no. 2, pp. 1084-1101, 2008.

[22] E. Tornatore, S. Buccellato, and P. Vetro, "Stability of a stochastic SIR system," Physica A, vol. 354, pp. 111-126, 2005.

[23] A. Gray, D. Greenhalgh, L. Hu, X. Mao, and J. Pan, "A stochastic differential equation SIS epidemic model," SIAM Journal on Applied Mathematics, vol. 71, no. 3, pp. 876-902, 2011.

[24] J. Yu, D. Jiang, and N. Shi, "Global stability of two-group SIR model with random perturbation," Journal of Mathematical Analysis and Applications, vol. 360, no. 1, pp. 235-244, 2009.

[25] E. Beretta, V. Kolmanovskii, and L. Shaikhet, "Stability of epidemic model with time delays influenced by stochastic perturbations," Mathematics and Computers in Simulation, vol. 45, no. 3-4, pp. 269-277, 1998.

[26] R. Z. Has'minskiĭ, Stochastic Stability of Differential Equations, vol. 7 of Monographs and Textbooks on Mechanics of Solids and Fluids: Mechanics and Analysis, Sijthoff \& Noordhoff, Alphen aan den Rijn, The Netherlands, 1980.

[27] D. J. Higham, "An algorithmic introduction to numerical simulation of stochastic differential equations," SIAM Review, vol. 43, no. 3, pp. 525-546, 2001.

[28] T. C. Gard, Introduction to Stochastic Differential Equations, vol. 114 of Monographs and Textbooks in Pure and Applied Mathematics, Marcel Dekker, New York, NY, USA, 1988.

[29] G. Strang, Linear Algebra and Its Applications, Thomson Learning, Glendale, Calif, USA, 1988.

[30] C. Zhu and G. Yin, "Asymptotic properties of hybrid diffusion systems," SIAM Journal on Control and Optimization, vol. 46, no. 4, pp. 1155-1179, 2007. 


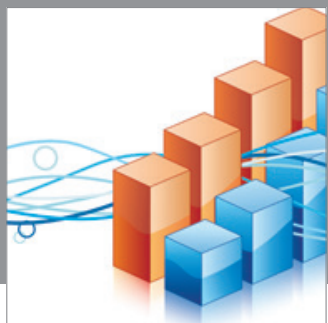

Advances in

Operations Research

mansans

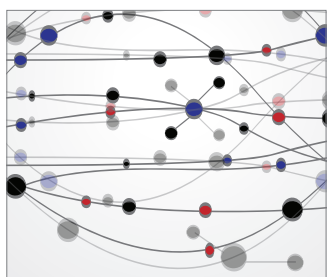

The Scientific World Journal
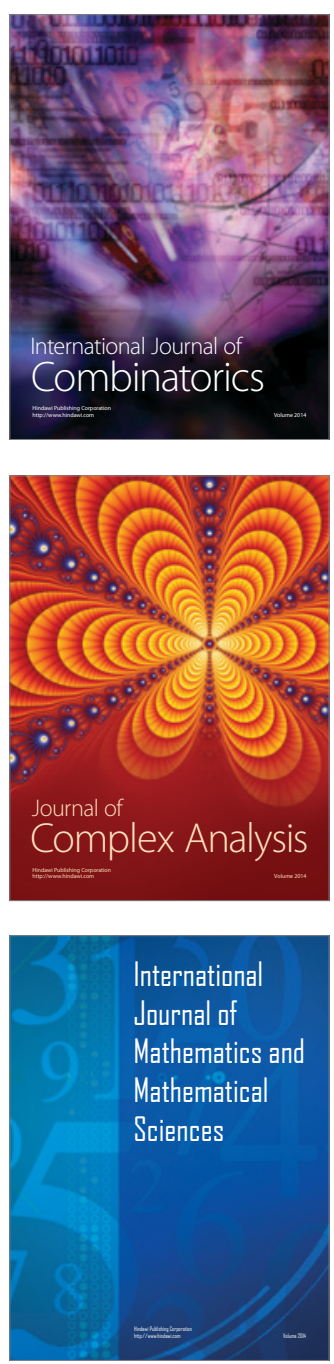
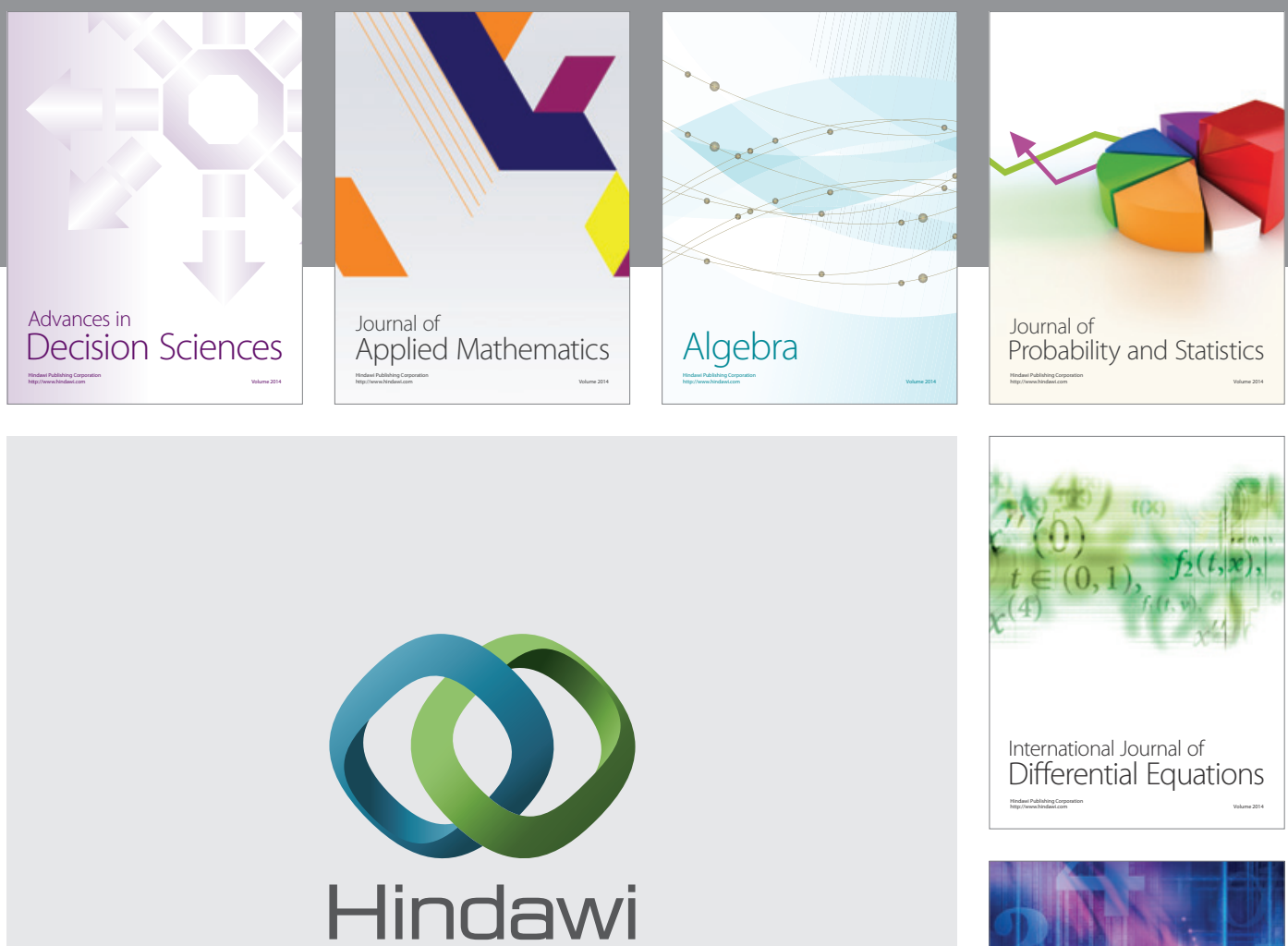

Submit your manuscripts at http://www.hindawi.com
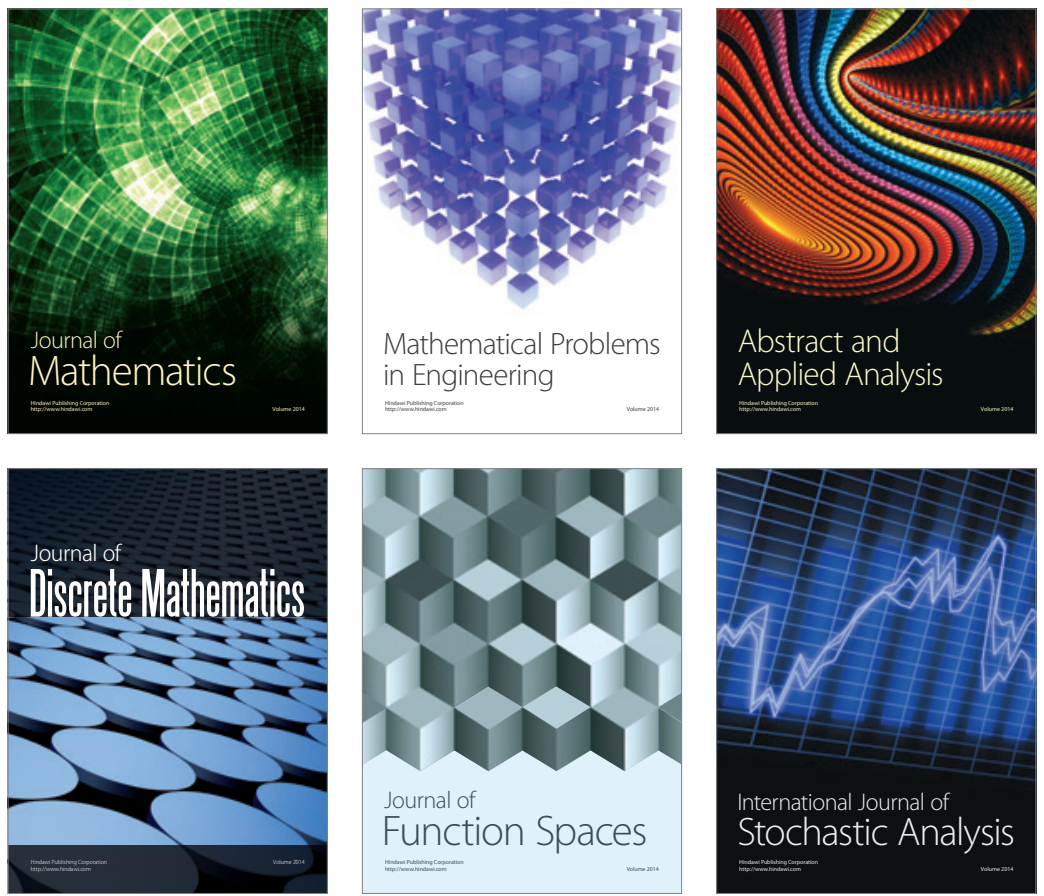

Journal of

Function Spaces



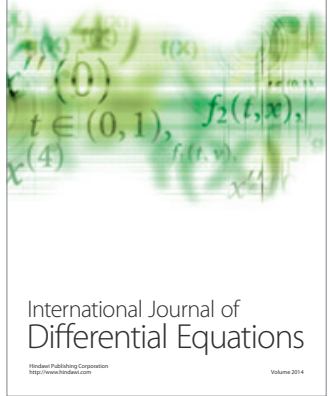
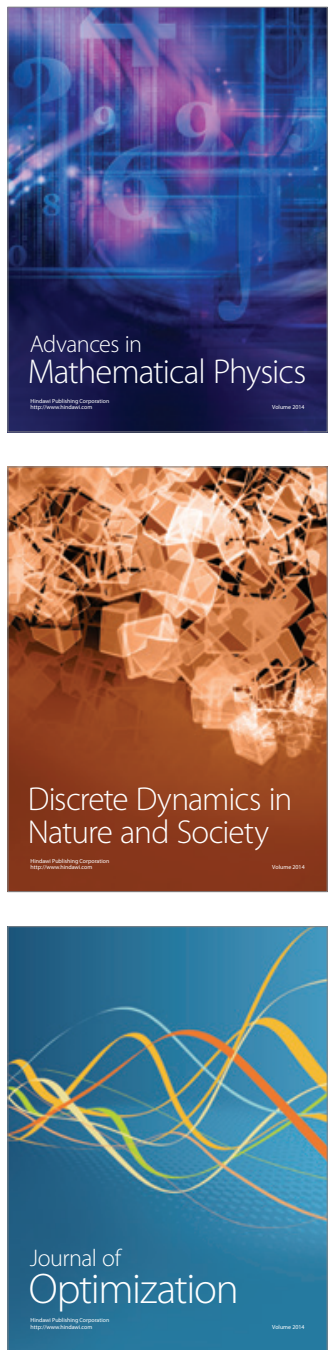